\title{
Facile Synthesis of Polypyrrole/Titanate Core-Shell Nanorods and Their Electrorheological Characteristics
}

\author{
Jiexu Zhang ${ }^{1}$, Ying $\mathrm{He}^{2}$, Lijun $\mathrm{Ji}^{1}$ \\ ${ }^{1}$ College of Chemical Engineering, East China University of Science and Technology, Shanghai, China; ${ }^{2}$ Key Laboratory for Ul- \\ trafine Materials of Ministry of Education, School of Materials Science and Engineering, East China University of Science and \\ Technology, Shanghai, China. \\ Email: rehey@ecust.edu.cn
}

Received November $9^{\text {th }}, 2010$; revised January $14^{\text {th }}, 2011$; accepted January $24^{\text {th }}, 2011$.

\begin{abstract}
In this work, polypyrrole (PPy)/titanate (TN) composite nanorods were successfully synthesized using cetyl trimethylammonium bromide (CTAB) as a structure-directing agent by in situ chemical oxidative polymerization. The structural characterization indicated that the new composite rods were core (TN)-shell (PPy) nanostructure with the average diameter in the range of 250-300 $\mathrm{nm}$. Further, this semiconducting composite can be used as a dispersed phase in silicone oil for a new electrorheological (ER) fluid, and its ER behavior was investigated under steady and oscillatory shear. It was found that the PPy/TN fluid showed typical ER characteristics under an external electric field.
\end{abstract}

Keywords: Polypyrrole, Titanate Nanorods, Conducting Composite, Electrorheological Fluid, Viscoelastic Properties

\section{Introduction}

In recent years, considerable efforts have been made towards the design and synthesis of one-dimensional (1D) nanostructured materials (e.g. nanotube, nanowire and nanorod) due to their potential applications in nanoscale electronic and photonic devices, as well as in biotechnology. More recently, interesting ER properties induced by $1 \mathrm{D}$ materials -based fluids have also attracted wide attention [1-3].

As one of the important smart materials, ER fluids have been intensively investigated over the past decades. ER fluids are commonly known as suspensions consisting of polarizable particles dispersed in an insulating liquid medium [4]. Their rheological properties can rapidly and reversibly change upon application of an electric field. This rapid and reversible electric-controlled mechanical response makes ER fluids to be used in the automotive industry for clutch, brake and damping systems [5]. To facilitate the practical application of ER technology, current research has focused on the development of optimal anhydrous ER materials with highperformance. Among these materials, 1D nanomaterials have had the advantage of the ER materials with a good
ER efficiency due to their large aspect ratio (large induced dipole moments) and good suspended stability. In particular, 1D conducting nanocomposites, containing inorganic nanotubes/nanowires coated with conducting polymer, provide an exciting system exhibiting improved chemical and physical properties over those of core or shell materials [6]. Since conducting polymers (e.g. polyaniline and polypyrrole) have been developed for ER application in the past years because of their high polarizability, good thermal stability and controllable conductivities and dielectric properties. Therefore, it is expected that 1D conducting nanocomposites with coreshell structure could be used as advanced ER fluids.

In this paper, we present a facile way to prepare a new conducting nanocomposite in which titanate $(\mathrm{TN})$ rods are encapsulated in the shell of polypyrrole (PPy). $\mathrm{TiO}_{2-}$ derived TN nanorods with wide band-gaps have also recently received increasing interest due to their important photocatalytic, photovoltaic, and semiconducting properties [7]. Hydrothermal treatment of $\mathrm{TiO}_{2}$ precursors in a highly alkaline medium is an effective way to the synthesis of 1D TN nanostructures. The PPy/TN composite nanorods were obtained by chemical oxidative polymerization directed by CTAB in the presence of TN. 
The structure and morphology of the nanorods were characterized. Furthermore, the potential application of $\mathrm{PPy} / \mathrm{TN}$ nanorods as an ER fluid under both steady and dynamic shear is also investigated, which might be helpful in the design and fabrication of ER materials with high-performance.

\section{Experimental}

\subsection{Synthesis of PPy/TN Composite Nanorods}

TN rods were prepared according to the report [8], with a minor modification. In a typical synthesis, 1.5 of $\mathrm{TiO}_{2}$ powder (Degussa P25, Germany) was mixed with $70 \mathrm{ml}$ of $10 \mathrm{M} \mathrm{NaOH}$ solution, which was followed by hydrothermal treatment of the mixture at $200^{\circ} \mathrm{C}$ in a $100 \mathrm{ml}$ Teflon-lined autoclave for $24 \mathrm{~h}$. The white precipitate was separated by filtration and washed with distilled water until $\mathrm{pH}$ was around 7 . The samples were then dried in an oven at $60^{\circ} \mathrm{C}$ for $12 \mathrm{~h}$.

To obtain PPy/TN composite nanorods, $0.5 \mathrm{~g}$ of TN nanorods were dispersed in $50 \mathrm{ml}$ of $0.1 \mathrm{M} \mathrm{CTAB}$ solution under ultrasonication at room temperature for $1 \mathrm{~h}$. After that, the mixture was cooled to $0-5^{\circ} \mathrm{C}$ with vigorous stirring. Then, a precooled solution of pyrrole monomer was introduced into the above-mentioned solution. After $0.5 \mathrm{~h}, 50 \mathrm{ml}$ of ammonium persulfate (APS) precooled solution was added dropwise to the mixture (molar ratio of Py/APS $=1$ ), and the polymerization reaction was allowed to proceed at $0-5^{\circ} \mathrm{C}$ for $24 \mathrm{~h}$. The resulting precipitate was filtered and washed with distilled water and acetone. Finally, the product was dried in a vacuum oven at $60^{\circ} \mathrm{C}$ for $24 \mathrm{~h}$.

\subsection{Characterization of Materials}

FT-IR spectra of the samples were obtained on Nicolet Magna-550 spectrometer in the range of $4000-400 \mathrm{~cm}^{-1}$, the morphology of the nanocomposite was studied using JEOL JSM-6360LV scanning electron microscope (SEM).

To prepare the ER fluid, PPy/TN particles were firstly dedoped by immersion in $3 \mathrm{vol} \%$ ammonia and then dried in a vacuum oven at $60^{\circ} \mathrm{C}$ for $12 \mathrm{~h}$. The dried particles were dispersed in silicone oil (Fluid 200, Dow Corning, UK; viscosity $\eta_{\mathrm{c}}=108 \mathrm{mPa} \cdot \mathrm{s}$, density $d_{\mathrm{c}}=$ $0.965 \mathrm{~g} / \mathrm{cm}^{3}$ ) to form the fluid concentration of $10 \mathrm{wt} . \%$. Measurements of rheological properties of the prepared fluids were carried out under controlled shear rate (CSR) mode using a coaxial cylinder viscometer (Bohlin GEMINI, Malvern Instruments, UK). The suspensions were placed in the Couette cell with the rotating inner cylinder of $14 \mathrm{~mm}$ in diameter and the outer cylinder separated by a $0.7 \mathrm{~mm}$ gap. They were connected to a DC power supply producing the following field strength: $E=0.5-3 \mathrm{kV} / \mathrm{mm}$. All the experiments were carried out at the temperature of $25^{\circ} \mathrm{C}$ in the shear rate range $0.1-700$ $\mathrm{s}^{-1}$. Static yield stress was obtained from measurements performed in CSS mode. Further, dynamic viscoelastic tests were performed through dynamic strain sweeps and frequency sweeps. The strain sweep was carried out with applied strains of $10^{-4}$ to 1.0 at a frequency of $10 \mathrm{~Hz}$ under an electric field in order to determine the linear viscoelastic region. The rheological parameters were then obtained from the frequency sweep tests $(0.1$ to $50 \mathrm{~Hz})$ at fixed strain amplitude in the linear viscoelastic region.

\section{Results and Discussion}

\subsection{Structure of Materials}

The chemical structure of PPy/TN composite nanorods was investigated using FT-IR spectroscopy. Figure 1 shows the IR spectra of the PPy and PPy/TN nanorods. It is evident that the composite shows similar FT-IR characteristic bands to those of polypyrrole in the 900-1600 $\mathrm{cm}^{-1}$ range, i.e., $1550,1460,1300$ and $1040 \mathrm{~cm}^{-1}$ bands, which are assigned to the pyrrole ring stretching, $\mathrm{C}-\mathrm{N}$ stretching mode, the in-plane vibrations of $\mathrm{C}-\mathrm{H}$, respectively [9]. In addition, a broad band at $3430 \mathrm{~cm}^{-1}$ is attributed to the $\mathrm{N}-\mathrm{H}$ stretching mode, and the bands at $1640 \mathrm{~cm}^{-1}$ correspond to the bending mode of $\mathrm{H}-\mathrm{O}-\mathrm{H}$. On the other hand, other bands at 914 and $465 \mathrm{~cm}^{-1}$ suggest the occurrence of the $\mathrm{TiO}_{6}$ octahedra [10]. The above results indicate that the as-synthesized composite contain both PPy and TN components.

The morphologies of TN and PPy/TN nanorods characterized by SEM are shown in Figure 2. The as-synthesized TN nanorods with diameters of about $200 \mathrm{~nm}$ and lengths of up to several micrometers are observed (Figure 2(a)). In contrast to them, PPy/TN composite exhibits larger diameter than that of TN, i.e. $250-300 \mathrm{~nm}$. PPy particles are almost deposited on the surface of $\mathrm{TN}$

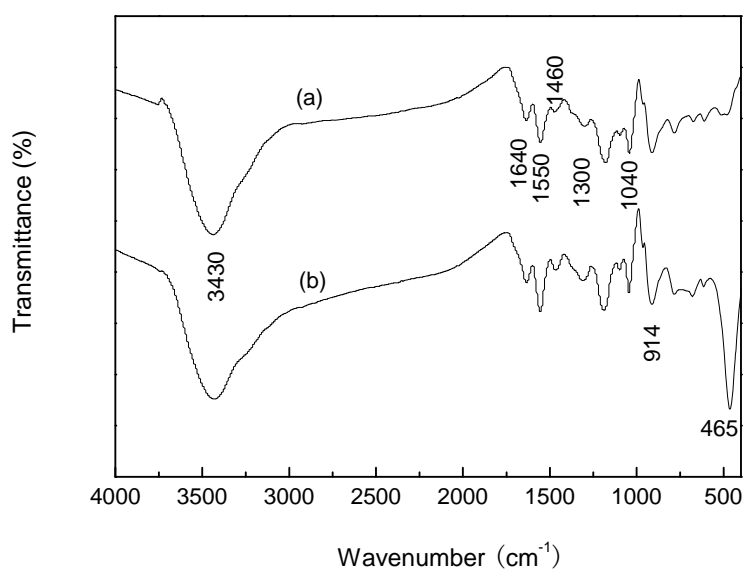

Figure 1. FT-IR spectra of (a) PPy and (b) PPy/TN composite nanorods. 


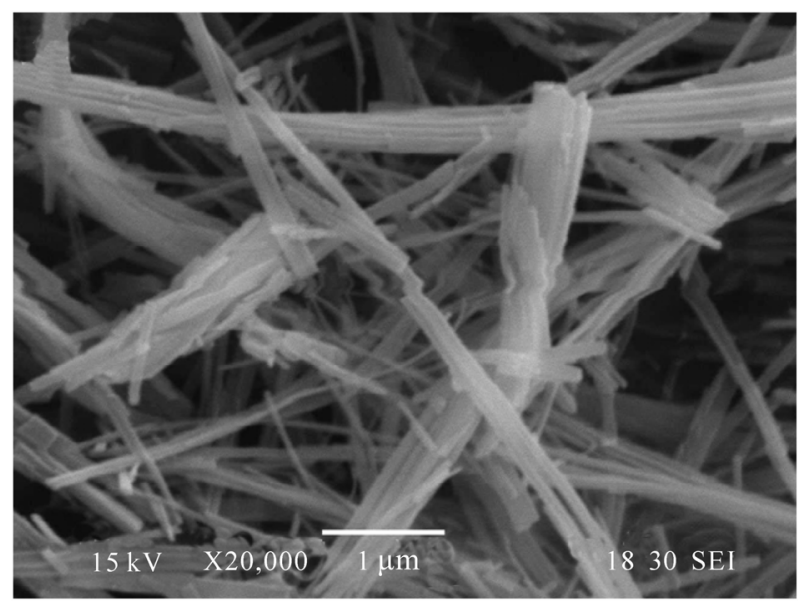

(a)

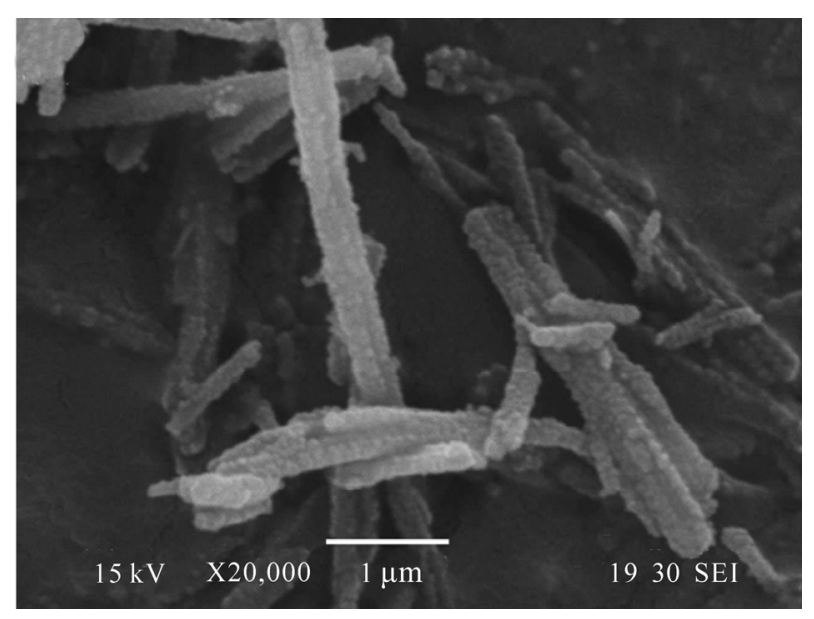

(b)

Figure 2. SEM images of (a) TN nanorods and (b) PPy/TN composite nanorods.

nanorods to form a core-shell structure shown in Figure 2(b). Also, the aspect ratios of PPy/TN nanorods are estimated to be 10-15.

\subsection{ER Behavior of PPy/TN Fluid}

This new core-shell nanocomposite can be used for ER application. Figure 3 provides the flow curve for PPy/ TN based ER fluid measured in CSR mode under four different electric field strengths. Without an electric field, the suspension shows a slight departure from Newtonian fluid behavior due to the relatively high particle concentration. When an electric field is applied to the suspension, the shear stress increases quickly with electric field, and yield stresses appear like in a Bingham fluid, which is the typical rheological characteristic of ER fluid under an electric field [11]. The figure also shows that, in the low-shear-rate region the shear stress slightly decreases with increasing shear rate up to a critical shear rate, and

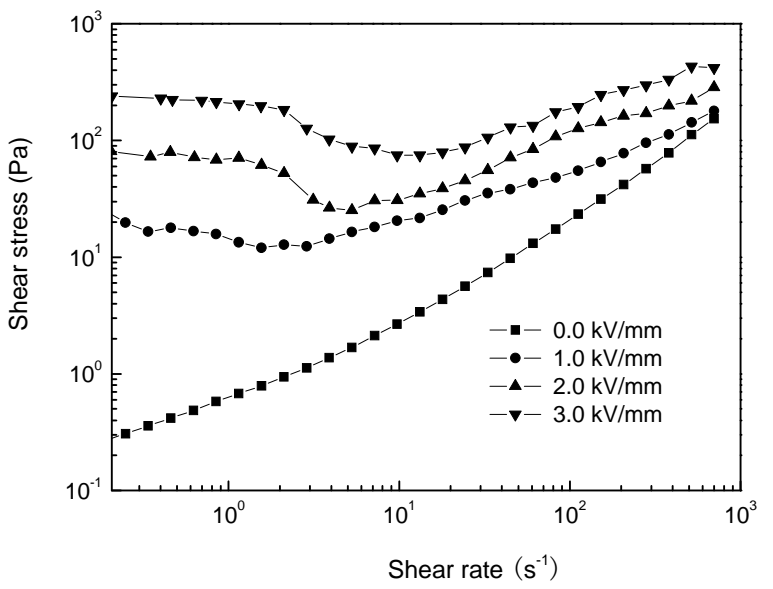

Figure 3. Shear stress versus shear rate for PPy/TN-based fluid under different electric field strengths.

then increases with shear rate. The critical shear rate is a transition point at which the fluid starts to exhibit pseudo-Newtonian behavior. This phenomenon is related to the structural change of ER fluid under shear. The dispersed particles are polarized and formed chain-like structures as a result of electrostatic forces generated between the dipoles induced by the external electric field. In the low-shear-rate region, with increasing shear rate the slight decrease of shear stress is a consequence of the destruction rate of chain-like structures exceeding their reformation rate. In the high-shear-rate region, the shear stress increases with shear rate showing pseudo-Newtonian, implying that the hydrodynamic forces rather than the electrostatic forces begins to dominate the flow behavior. This is similar to other reports $[12,13]$. Further, one can note that the critical shear rate shifts to high value with increasing electric fields, which is related to the magnitude of attractive interactions between the dispersed particles.

The change of the microstructure of PPy/TN composite fluid can also be detected under dynamic shear. Figure 4(a) shows the storage modulus $\left(G^{\prime}\right)$ and loss modulus $\left(G^{\prime \prime}\right)$ as a function of strain for $10 \mathrm{wt} . \%$ PPy/TN fluid. It can be found that both moduli increase with increasing electric field strength and $G^{\prime}$ is larger than $G^{\prime \prime}$ in the linear viscoelastic range. This is mainly caused by the elasticity of PPy/TN based fluid. The chain-like structures formed by the polarized PPy/TN particles become more elastic and stiffer at higher electric field, which makes the fluid behave like solid, resulting in a larger $G^{\prime}$. When the strain is increased, for example, exceeds the critical strain, the chain-like structures will collapse and the fluid shows liquid behavior, i.e. $G^{\prime \prime}>G^{\prime}$. Figure 4(b) presents $G^{\prime}$ and $G^{\prime \prime}$ of PPy/TN fluid as a function of frequency at a small strain of $2 \times 10^{-4}$ in the linear viscoelas- 


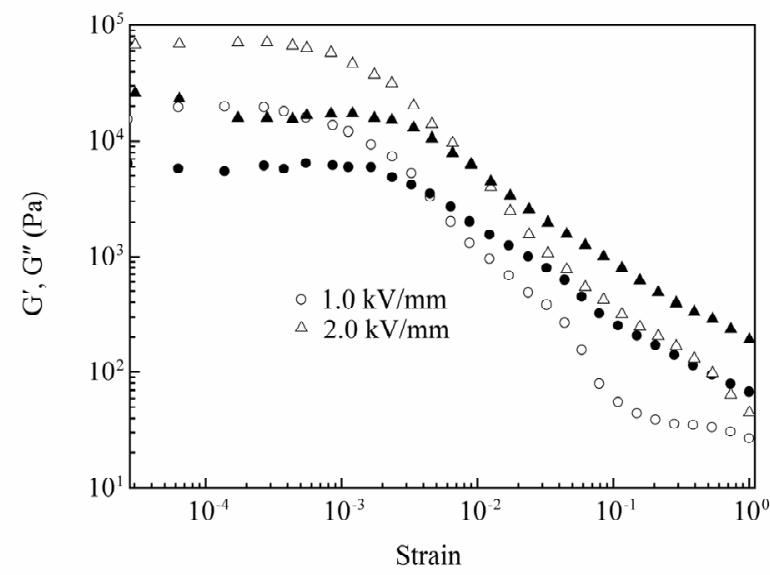

(a)

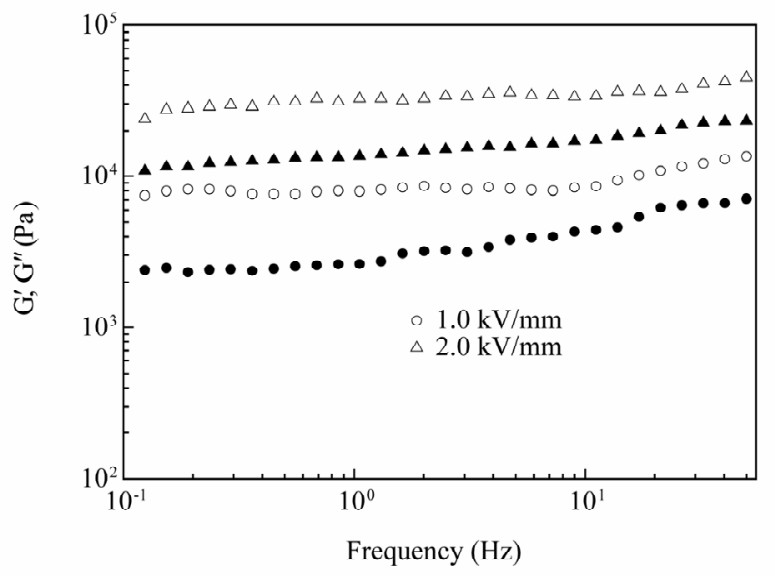

(b)

Figure 4. $G^{\prime}$ and $G^{\prime \prime}$ as a function of strain amplitude (a) and (b) frequency for PPy/TN fluid under different electric field strengths: open symbols for $G^{\prime}$; shaded symbols for $G^{\prime \prime}$.

tic range. At the same electric field strength, both moduli are almost independent of frequency in the low frequency range due to rigid chain-like structure, while with increasing frequency they increase with frequency showing a transition to a nonlinear region because of the onset of chain rupture $[14,15]$.

\section{Conclusions}

A new polypyrrole/titanate composite nanorod was synthesized via chemical oxidative polymerization of pyrrole directed by cetyl trimethylammonium bromide in the presence of titanate nanorods. FTIR and SEM characterization confirmed that polypyrrole was deposited on the surface of titanate particles and formed a core-shell structure. Further, it was observed that the suspension of polypyrrole/titanate exhibited typical ER behavior under an external electric field. Shear stress and dynamic moduli increase with electric field due to elasticity of the ER fluid. And in the linear viscoelastic range, storage modulus is larger than loss modulus under an electric field.

\section{REFERENCES}

[1] J. B. Ying and X. P. Zhao, "Titanate Nano-Whisker Electrorheological Fluid with High Suspended Stability and ER Activity," Nanotechnology, Vol. 17, No. 1, 2006, pp. 192-196. doi:10.1088/0957-4484/17/1/031

[2] Y. D. Liu, F. F. Fang and H. J. Choi, "Silica Nanoparticle Decorated Conducting Polyaniline Fibers and Their Electrorheology," Materials Letters, Vol. 64, No. 2, 2010, pp. 154-156. doi:10.1016/j.matlet.2009.10.031

[3] Y. Cheng, K. Wu, F. Liu, J. Guo, X. Liu, G. Xu and P.
Cui, "Facile Approach to Large-Scale Synthesis of 1D Calcium and Titanium Precipitate (CTP) with High Electrorheological Activity," ACS Applied Materials \& Interfaces, Vol. 2, No. 3, 2010, pp. 621-625. doi:10.1021/am900841m

[4] H. Block and J. P. Kelly, "Electro-Rheology," Journal of Physics D: Applied Physics, Vol. 21, No. 12, 1998, pp. 1661-1667. doi:10.1088/0022-3727/21/12/001

[5] T. Hao, "Electrorheological Fluids," Advanced Materials, Vol. 13, No. 24, 2001, pp. 1847-1857. doi:10.1002/1521-4095(200112)

[6] Q. Cheng, Y. He, V. Pavlinek, C. Li and P. Saha, "Surfactant-Assisted Polypyrrole/Titanate Composite Nanofibers: Morphology, Structure and Electrical Properties," Synthetic Metals, Vol. 158, No. 21-24, 2008, pp. 953-957. doi:10.1016/j.synthmet.2008.06.022

[7] D. V. Bavykin, J. M. Friedrich and F. C. Walsh, "Protonated Titanates and $\mathrm{TiO}_{2}$ Nanostructured Materials: Synthesis, Properties, and Applications," Advanced Materials, Vol. 18, No. 21, 2006, pp. 2807-2824. doi:10.1002/adma.200502696

[8] Y. Lan, X. P. Gao, H. Y. Zhu, Z. F. Zheng, T. Y. Yan, F. Wu, S. P. Ringer and D. Y. Song, "Titanate Nanotubes and Nanorods Prepared from Rutile Powder," Advanced Functional Materials, Vol. 15, No. 8, 2005, pp. 1310-1318. doi:10.1002/adfm.200400353

[9] G. I. Mathys and V. T. Truong, "Spectroscopic Study of Thermo-Oxidative Degradation of Polypyrrole Powder by FT-IR," Synthetic Metals, Vol. 89, No. 2, 1997, pp. 103-109. doi:10.1016/S0379-6779(98)80122-7

[10] O. Harizanov, A. Harizanova and T. Ivanova, "Formation and Characterization of Sol-Gel Barium Titanate," Materials Science and Engineering: B, Vol. 106, No. 2, 2004, pp. 191-195. doi:10.1016/i.mseb.2003.09.014

[11] Y. T. Lim, J. H. Park and O. O. Park, "Improved Elec- 
trorheological Effect in Polyaniline Nanocomposite Suspensions," Journal of Colloid and Interface Science, Vol. 245, No. 1, 2002, pp. 198-203. doi:10.1006/jis. 2001.7983

[12] H.-J. Choi, M.-S. Cho and K. To, "Electrorheological and Dielectric Characteristics of Semiconductive PolyanilineSilicone Oil Suspensions," Physica A: Statistical Mechanics and Its Applications, Vol. 254, No. 1-2, 1998, pp. 272-279.

doi:10.1016/S0378-4371(98)00005-3

[13] Q. Cheng, V. Pavlinek, A. Lengalova, C. Li, T. Belza and P. Saha, "Electrorheological Properties of New Mesoporous Material with Conducting Polypyrrole in Mesoporous silica," Microporous and Mesoporous Materials, Vol. 94, No. 1-3, 2006, pp. 193-199.

doi:10.1016/j.micromeso.2006.03.039

[14] S.-G. Kim, J.-W. Kim, M.-S. Cho, H.-J. Choi and M.-S. Jhon, "Viscoelastic Characterization of Semiconducting Dodecylbenzenesulfonic Acid Doped Polyaniline Electrorheological Suspensions," Journal of Applied Polymer Science, Vol. 79, No. 1, 2001, pp. 108-114. doi:10.1002/1097-4628(20010103)

[15] M.-S. Cho, H.-J. Choi and W.-S. Ahn, "Enhanced Electrorheology of Conducting Polyaniline Confined in MCM41 Channels," Langmuir, Vol. 20, No. 1, 2004, pp. 202-207. doi:10.1021/la035051z 